\title{
The Dentists' View of Dental Hygienist Duties in Korea
}

\author{
Yang-Keum Han ${ }^{1}$, Ji-Su Yu², Seung-Hee Kim³ ${ }^{3}$, Jin-Young Yang ${ }^{4}$, Soo-Myoung Bae ${ }^{5}$, and \\ Soo-Jeong Hwang ${ }^{6, \dagger}$ \\ 'Department of Dental Hygiene, Daejeon Health Institute of Technology, Daejeon 34504, \\ ${ }^{2}$ Department of Dental Hygiene, Gumi University, Gumi 39213, \\ ${ }^{3}$ Department of Dental Hygiene, Gwangju Health University, Gwangju 62287, \\ ${ }^{4}$ Department of Dental Hygiene, Daejeon Institute of Science and Technology, Daejeon 35408, \\ ${ }^{5}$ Department of Dental Hygiene, College of Dentistry, Research Institute of Oral Science, Gangneung-Wonju \\ National University, Gangneung 25457, \\ ${ }^{6}$ Department of Dental Hygiene, College of Medical Science, Konyang University, Daejeon 35365, Korea
}

\begin{abstract}
Background: The number and curriculum of dental hygienists in Korea have dramatically increased. Controversies have since resulted from insufficient job descriptions of the work performed by a dental hygienist. A dentist's perception was examined to legally reflect the actual work of dental hygienists.

Methods: Four hundred and nineteen dentists were surveyed about the duties of a dental hygienist. Their views on the career and availability of each job were examined. The duties of the dental hygienist include 13 items in dental treatment preparation, 14 items of radiography, 21 items of preventive dentistry, 6 items of periodontal treatment, 12 items of oral medicine, 12 items of conservative dentistry, 8 items of prosthetics, 10 items of orthodontics, 7 items of oral and maxillofacial surgery, 6 items of implantation, 6 items of impression taking and model fabrication, 5 items of anesthesia and injection, 11 items of management and administrative, and 3 items of self-development.

Results: Most of the duties were doable by a dental hygienist. Many dentists reported that managing implants, oral hygiene of special patients, some duties in oral medicine, teeth brightening, making temporary crowns, making individual trays, selecting shades, ligaturing, and precision impressions need $\geq 3$ years of experience. Duties perceived by dentists not to be performed by dental hygienists were reading radiographs (55.4\%), suture and stitch out (48.0\%), intramuscular injection (36.0\%), root planning (27.2\%), cementation and removal of prostheses (23.2\%), and examining pulp vitality (22.0\%) .

Conclusion: Current laws are to be revised to include, the care provided by dental hygienists and under a physician's supervision. Flexibility is also needed to cope with rapidly changing dental technology.
\end{abstract}

Key Words: Dental hygienists, Dentists, Task performance and analysis

\section{Introduction}

Korean dental hygienist education, which began in 1965, has improved for the past 50 years; it has been organized into a three-year professional and a four-year Bachelor's degree curriculum. In 2020, the number of licensed dental hygienists reached 89,993. In addition, the quality of education improved after a Master and Doctorate degrees in dental hygiene were initiated. In order to enhance the professionalism and job of dental hygienists, the dental hygiene education community has been working to establish new educational goals and standardized curricula ${ }^{1,2)}$.

Despite the development of the education system for dental hygienists, controversies over their duties continue. Dental hygienists should perform only the tasks specified in the Act for medical technicians. However, Korean dental hygienists perform medical and dental support 
services in addition to those specified under the law and in addition to those done under the direction and supervision of a dentist. Since these are not specified in the law, the criteria for judging the legitimacy of the relevant actions are ambiguous, and in fact, the number of administrative determinations has increased ${ }^{3)}$. In addition, the inconsistency between the duty actually performed by the dental hygienist at the dental clinic or hospital and that performed by the dental hygienist prescribed in the law can lead to an increase in work stress and turnover in the dental field ${ }^{4-7)}$.

Korean dentists and hygienists are experiencing chronic labor shortages ${ }^{3)}$. Thus, most private dentists require that a dental hygienist be able to perform basic dental care preparation, consultation and guides for patients, perform intra- or extra-oral $x$-ray imaging, and provide preventive dental care and routine chair-side dental assistance ${ }^{8)}$. However, there are objections to this from academic professors and dentists who oppose such transfer of duties to the hands of dental hygienists. This disagreement can be a barrier for revising the existing law regarding the role of dental hygienists in practice. In addition, previous research has reported that the role of dental hygienists is not systematic, and therefore, there is a lack of awareness on the part of dentists regarding the scope of a dental hygienist duty ${ }^{8-11)}$. Due to insufficient legal description of the role of dental hygienists, potential conflicts between patients and dentists are bound to occur. Furthermore, in some cases, the Ministry of Health and Welfare had to obtain a vote on defining the role of dental hygienists. The purpose of this study was to examine the opinions of dentists on the role of dental hygienists. The data obtained can be used as a basis to enact revisions to the existing laws on the role of dental hygienists.

\section{Materials and Methods}

\section{Research subject}

We planned to investigate about 500 dentists through stratified sampling by region and institution. Because it was difficult to get the cooperation of the society for dentists, we proceeded with the convenience extraction method by using seven researchers who included three dentists and four dental hygienists. Korea was divided into the metropolitan area, Gyeongsang area, Jeolla area, Chungcheong area, and Gangwon area, and designated each area. The initial survey method was e-mail; however, the response rate by researchers was only 3 to $8 \%$. Then, face to face surveys were added, and the response rate went up to about 30 to $40 \%$. The total number of response was 419 . The general characteristics are shown in Table 1. The questionnaire was identified as selecting non-response items that were considered difficult for personal in-

Table 1. General Characteristics of the Dentist Studied

\begin{tabular}{|c|c|c|}
\hline Variable & Category & $\mathrm{n}(\%)$ \\
\hline \multirow[t]{6}{*}{ Working place } & College hospital & $26(6.2)$ \\
\hline & General hospital & $16(3.8)$ \\
\hline & Private dental hospital & $45(10.7)$ \\
\hline & Dental clinic & $324(77.3)$ \\
\hline & Public health center & $2(0.5)$ \\
\hline & No response & $6(1.4)$ \\
\hline \multirow[t]{3}{*}{ Sex } & Male & $333(79.5)$ \\
\hline & Female & 79 (18.9) \\
\hline & No response & $7(1.7)$ \\
\hline \multirow[t]{4}{*}{ Position } & Owner & $286(68.3)$ \\
\hline & $\begin{array}{l}\text { Employee of private dental clinic } \\
\text { or hospital }\end{array}$ & $65(15.5)$ \\
\hline & $\begin{array}{l}\text { Official or employee of college } \\
\text { or general hospital }\end{array}$ & $41(9.8)$ \\
\hline & No response & $27(6.4)$ \\
\hline \multirow[t]{5}{*}{ Age } & $20 \mathrm{~s}$ & $20(4.8)$ \\
\hline & $30 \mathrm{~s}$ & $81(19.3)$ \\
\hline & $40 \mathrm{~s}$ & $114(27.2)$ \\
\hline & 50 years old or older & $132(31.5)$ \\
\hline & No response & $72(17.2)$ \\
\hline \multirow[t]{4}{*}{ Area } & Metropolitan city & $252(60.1)$ \\
\hline & Small cities & $143(34.1)$ \\
\hline & Rural area & $9(2.1)$ \\
\hline & No response & $15(3.6)$ \\
\hline \multirow[t]{6}{*}{ Location } & Seoul, Incheon, Gyeonggi area & $161(38.4)$ \\
\hline & Gyeongsang area & $39(9.3)$ \\
\hline & Jeolla area & $44(10.5)$ \\
\hline & Chungcheong area & $115(27.4)$ \\
\hline & Gangwon area & $32(7.6)$ \\
\hline & No response & $28(6.7)$ \\
\hline \multirow{3}{*}{$\begin{array}{l}\text { Specialist } \\
\text { training }\end{array}$} & Yes & $185(44.2)$ \\
\hline & No & $210(50.1)$ \\
\hline & No response & $24(5.7)$ \\
\hline \multirow[t]{4}{*}{ Degree } & Bachelor & $121(28.9)$ \\
\hline & Master & $143(34.1)$ \\
\hline & Doctor & $132(31.5)$ \\
\hline & No response & $23(5.5)$ \\
\hline Total & & 419 (100) \\
\hline
\end{tabular}


formation or judgment, and no response was included in the analysis.

\section{Questionnaire content}

The survey was conducted on the duties of dental hygienists extracted from the 2nd job analysis of dental hygienists at the Korea National Institute of Health in $2012^{12)}$. The dental hygienist's duties include dental treatment preparation, radiography, preventive dentistry, oral medicine, periodontal treatment, conservative dentistry, prosthetics, orthodontics, oral and maxillofacial surgery, implantation, impression taking and model, anesthesia and injection, management and administrative, and selfdevelopment. Some jobs had overlapping classifications, making the description vague. In order to strictly follow the classification of the 2 nd job analysis of the dental hygienist, the classification was used for the investigation without modification according to the intention of this researcher. The response was to write on whether dental hygienists could perform each duty and how much experience was required. We surveyed the necessary clinical experience for duty performance because it could

Table 2. Dentist's View of the Duties of Dental Hygienists in Dental Treatment Preparation

\begin{tabular}{|c|c|c|c|c|c|c|}
\hline \multirow[b]{2}{*}{ Duty } & \multicolumn{3}{|c|}{ Clinical experience required } & \multirow{2}{*}{$\begin{array}{l}\text { Possible in } \\
\text { the future }\end{array}$} & \multirow[b]{2}{*}{ Impossible } & \multirow{2}{*}{$\begin{array}{c}\text { No } \\
\text { response }\end{array}$} \\
\hline & $\begin{array}{l}\text { Beginners } \\
\text { available }\end{array}$ & $\begin{array}{c}3 \text { years or } \\
\text { more }\end{array}$ & $\begin{array}{l}5 \text { years or } \\
\text { more }\end{array}$ & & & \\
\hline Receiving patients & $314(74.9)$ & $71(16.9)$ & $21(5.0)$ & $1(0.2)$ & $8(1.9)$ & $4(1.0)$ \\
\hline Adjusting position of patients & 309 (73.7) & $89(21.2)$ & $15(3.6)$ & $1(0.2)$ & $4(1.0)$ & $1(0.2)$ \\
\hline Dealing with infectious diseases & $238(56.8)$ & $142(33.9)$ & $23(5.5)$ & $4(1.0)$ & $9(2.1)$ & $3(0.7)$ \\
\hline Managing clinical clothes & $335(80.0)$ & $63(15.0)$ & $12(2.9)$ & $1(0.2)$ & $3(0.7)$ & $5(1.2)$ \\
\hline Personal protection & $305(72.8)$ & $91(21.7)$ & $18(4.3)$ & $1(0.2)$ & $3(0.7)$ & $1(0.2)$ \\
\hline Managing preoperative operators and assistants & $259(61.8)$ & $133(31.7)$ & $20(4.8)$ & $1(0.2)$ & $3(0.7)$ & $3(0.7)$ \\
\hline Managing preoperative patients & $250(59.7)$ & $144(34.4)$ & $19(4.5)$ & $1(0.2)$ & $3(0.7)$ & $2(0.5)$ \\
\hline Preventing equipment infection & $336(80.2)$ & $73(17.4)$ & $8(1.9)$ & $1(0.2)$ & $0(0.0)$ & $1(0.2)$ \\
\hline Disinfection & $381(90.9)$ & $31(7.4)$ & $5(1.2)$ & $1(0.2)$ & $0(0.0)$ & $1(0.2)$ \\
\hline Sterilizing the operating room & 309 (73.7) & $92(22.0)$ & $13(3.1)$ & $3(0.7)$ & $1(0.2)$ & $1(0.2)$ \\
\hline Using the sterilizer & $328(78.3)$ & $77(18.4)$ & $10(2.4)$ & $1(0.2)$ & $2(0.5)$ & $1(0.2)$ \\
\hline Using chemical disinfectants & $324(77.3)$ & $76(18.1)$ & $12(2.9)$ & $0(0.0)$ & $4(1.0)$ & $3(0.7)$ \\
\hline Disposal of medical waste & $330(78.8)$ & 78 (18.6) & $9(2.1)$ & $0(0.0)$ & $1(0.2)$ & $1(0.2)$ \\
\hline
\end{tabular}

Table 3. Dentist's View of the Duties of Dental Hygienists in Radiography

\begin{tabular}{|c|c|c|c|c|c|c|}
\hline \multirow[b]{2}{*}{ Duty } & \multicolumn{3}{|c|}{ Clinical experience required } & \multirow[b]{2}{*}{$\begin{array}{l}\text { Possible in } \\
\text { the future }\end{array}$} & \multirow[b]{2}{*}{ Impossible } & \multirow[b]{2}{*}{ No response } \\
\hline & $\begin{array}{l}\text { Beginners } \\
\text { available }\end{array}$ & $\begin{array}{c}3 \text { years or } \\
\text { more }\end{array}$ & $\begin{array}{c}5 \text { years or } \\
\text { more }\end{array}$ & & & \\
\hline Preparing for intraoral radiography & $394(94.0)$ & $20(4.8)$ & $2(0.5)$ & $1(0.2)$ & $1(0.2)$ & $1(0.2)$ \\
\hline Taking peri-apical radiography & $373(89.0)$ & $37(8.8)$ & $2(0.5)$ & $1(0.2)$ & $5(1.2)$ & $1(0.2)$ \\
\hline Taking bite-wing radiography & $323(77.1)$ & $73(17.4)$ & $5(1.2)$ & $1(0.2)$ & $13(3.1)$ & $4(1.0)$ \\
\hline Taking occlusal radiography & $306(73.0)$ & $84(20.0)$ & $8(1.9)$ & $1(0.2)$ & $15(3.6)$ & $5(1.2)$ \\
\hline Taking radiography of special patients & $184(43.9)$ & $167(39.9)$ & $33(7.9)$ & $3(0.7)$ & $20(4.8)$ & $12(2.9)$ \\
\hline Localization radiography & $226(53.9)$ & $136(32.5)$ & $20(4.8)$ & $3(0.7)$ & $17(4.1)$ & $17(4.1)$ \\
\hline Preparing for extraoral radiography & $369(88.1)$ & $33(7.9)$ & $3(0.7)$ & $0(0.0)$ & $7(1.7)$ & $7(1.7)$ \\
\hline Taking panorama radiography & $364(86.9)$ & $37(8.8)$ & $4(1.0)$ & $1(0.2)$ & $12(2.9)$ & $1(0.2)$ \\
\hline Taking cephalometric radiography & $274(65.4)$ & $78(18.6)$ & $10(2.4)$ & $6(1.4)$ & $36(8.6)$ & $15(3.6)$ \\
\hline Taking computed tomography & $241(57.5)$ & $85(20.3)$ & $15(3.6)$ & $16(3.8)$ & $48(11.5)$ & $14(3.3)$ \\
\hline Developing films & $358(85.4)$ & $26(6.2)$ & $2(0.5)$ & $0(0.0)$ & $16(3.8)$ & $17(4.1)$ \\
\hline Managing radiographs & $367(87.6)$ & $30(7.2)$ & $3(0.7)$ & $0(0.0)$ & 8 (1.9) & $11(2.6)$ \\
\hline Dealing with digital radiation devices & $323(77.1)$ & $72(17.2)$ & $8(1.9)$ & $1(0.2)$ & $6(1.4)$ & $9(2.1)$ \\
\hline Reading radiographs & $51(12.2)$ & $69(16.5)$ & $50(11.9)$ & $12(2.9)$ & $232(55.4)$ & $5(1.2)$ \\
\hline
\end{tabular}


represent insufficient training in the curriculum of dental hygiene or indicate the necessity of conservative education due to each task' difficulty and professional dental hygienist system. We showed the overall opinion of dentists about the duties of dental hygienists. The data was entered into
Excel 2010 (Microsoft, Redmond, WA, USA) and frequency analysis was performed.

Table 4. Dentist's View of the Duties of Dental Hygienists in Oral Disease Prevention

\begin{tabular}{|c|c|c|c|c|c|c|}
\hline \multirow[b]{2}{*}{ Duty } & \multicolumn{3}{|c|}{ Clinical experience required } & \multirow{2}{*}{$\begin{array}{l}\text { Possible in } \\
\text { the future }\end{array}$} & \multirow[b]{2}{*}{ Impossible } & \multirow[b]{2}{*}{ No response } \\
\hline & $\begin{array}{l}\text { Beginners } \\
\text { available }\end{array}$ & $\begin{array}{c}3 \text { years or } \\
\text { more }\end{array}$ & $\begin{array}{l}5 \text { years or } \\
\text { more }\end{array}$ & & & \\
\hline Charting preventive treatment records & $291(69.5)$ & $97(23.2)$ & $7(1.7)$ & $5(1.2)$ & $14(3.3)$ & $5(1.2)$ \\
\hline Examining dental plaque & $279(66.6)$ & $106(25.3)$ & $8(1.9)$ & $3(0.7)$ & $18(4.3)$ & $5(1.2)$ \\
\hline Examining oral bacteria & $223(53.2)$ & $109(26.0)$ & $13(3.1)$ & $9(2.1)$ & $60(14.3)$ & $5(1.2)$ \\
\hline Examining dental caries activity & $226(53.9)$ & $119(28.4)$ & $16(3.8)$ & $4(1.0)$ & $46(11.0)$ & $8(1.9)$ \\
\hline Managing oral hygiene by subject & $263(62.8)$ & $127(30.3)$ & $7(1.7)$ & $3(0.7)$ & $10(2.4)$ & $9(2.1)$ \\
\hline Professional tooth brushing & $245(58.5)$ & $129(30.8)$ & $16(3.8)$ & $2(0.5)$ & $21(5.0)$ & $6(1.4)$ \\
\hline Managing hypersensitivity & $229(54.7)$ & $125(29.8$ & $22(5.3)$ & $5(1.2)$ & $34(8.1)$ & $4(1.0)$ \\
\hline Flouride application & $326(77.8)$ & $74(17.7)$ & $12(2.9)$ & $2(0.5)$ & $2(0.5)$ & $3(0.7)$ \\
\hline Sealants & $221(52.7)$ & $127(30.3)$ & $34(8.1)$ & $8(1.9)$ & $26(6.2)$ & $3(0.7)$ \\
\hline Smoking control & $227(54.2)$ & $108(25.8)$ & $25(6.0)$ & $16(3.8)$ & $38(9.1)$ & $5(1.2)$ \\
\hline Consulting diet & $235(56.1)$ & $108(25.8)$ & $24(5.7)$ & $14(3.3)$ & $33(7.9)$ & $5(1.2)$ \\
\hline Managing eating or swallowing disorder & $181(43.2)$ & $129(30.8)$ & $31(7.4)$ & $16(3.8)$ & $51(12.2)$ & $11(2.6)$ \\
\hline Oral muscular exercise training & $243(58.0)$ & $111(26.5)$ & $15(3.6)$ & $12(2.9)$ & $25(6.0)$ & $13(3.1)$ \\
\hline Managing multiple dental caries & $167(39.9)$ & $161(38.4)$ & $42(10.0)$ & $10(2.4)$ & $32(7.6)$ & $7(1.7)$ \\
\hline Scaling & $336(80.2)$ & $61(14.6)$ & $10(2.4)$ & $2(0.5)$ & $5(1.2)$ & $5(1.2)$ \\
\hline Using manual scaler & $268(64.0)$ & $118(28.2)$ & $17(4.1)$ & $3(0.7)$ & $10(2.4)$ & $3(0.7)$ \\
\hline Using ultrasonic scaler & $347(82.8)$ & $55(13.1)$ & $9(2.1)$ & $1(0.2)$ & $3(0.7)$ & $4(1.0)$ \\
\hline Managing implant & $134(32.0)$ & $191(45.6)$ & $40(9.5)$ & $10(2.4)$ & $39(9.3)$ & $5(1.2)$ \\
\hline Oral hygiene of special patients & $149(35.6)$ & $188(44.9)$ & $56(13.4)$ & $5(1.2)$ & $13(3.1)$ & $8(1.9)$ \\
\hline Oral prophylaxis & $279(66.6)$ & $109(26.0)$ & $13(3.1)$ & $1(0.2)$ & $12(2.9)$ & $5(1.2)$ \\
\hline Managing scaler or curette & $246(58.7)$ & $122(29.1)$ & $23(5.5)$ & $2(0.5)$ & $20(4.8)$ & $6(1.4)$ \\
\hline
\end{tabular}

Table 5. Dentist's View of the Duties of Dental Hygienists in Oral Medicine

\begin{tabular}{|c|c|c|c|c|c|c|}
\hline \multirow[b]{2}{*}{ Duty } & \multicolumn{3}{|c|}{ Clinical experience required } & \multirow[b]{2}{*}{$\begin{array}{l}\text { Possible in the } \\
\text { future }\end{array}$} & \multirow[b]{2}{*}{ Impossible } & \multirow[b]{2}{*}{ No response } \\
\hline & $\begin{array}{l}\text { Beginners } \\
\text { available }\end{array}$ & $\begin{array}{c}3 \text { years or } \\
\text { more }\end{array}$ & $\begin{array}{c}5 \text { years or } \\
\text { more }\end{array}$ & & & \\
\hline Managing the TMD patients & $127(30.3)$ & $176(42.0)$ & $37(8.8)$ & $13(3.1)$ & $54(12.9)$ & $12(2.9)$ \\
\hline Physical therapy on head and neck & $165(39.4)$ & $135(32.2)$ & $35(8.4)$ & $19(4.5)$ & $53(12.6)$ & $12(2.9)$ \\
\hline Hot pack on head and neck & $200(47.7)$ & $120(28.6)$ & $27(6.4)$ & $19(4.5)$ & $39(9.3)$ & $14(3.3)$ \\
\hline Ultrasonic therapy on head and neck & $157(37.5)$ & $123(29.4)$ & $30(7.2)$ & $16(3.8)$ & $78(18.6)$ & $15(3.6)$ \\
\hline Exercise training of TMD patients & $151(36.0)$ & $147(35.1)$ & $35(8.4)$ & $17(4.1)$ & $51(12.2)$ & $18(4.3)$ \\
\hline Managing bruxism & $138(32.9)$ & $151(36.0)$ & $40(9.5)$ & $20(4.8)$ & $57(13.6)$ & $13(3.1)$ \\
\hline Managing snore & $131(31.3)$ & $151(36.0)$ & $40(9.5)$ & $18(4.3)$ & $65(15.5)$ & $14(3.3)$ \\
\hline Managing oral soft tissue disease & $124(29.6)$ & $147(35.1)$ & $42(10.0)$ & $16(3.8)$ & $74(17.7)$ & $16(3.8)$ \\
\hline Managing dry mouth & $132(31.5)$ & $154(36.8)$ & $38(9.1)$ & $15(3.6)$ & $63(15.0)$ & $17(4.1)$ \\
\hline Treating bad breath & 159 (37.9) & $132(31.5)$ & $35(8.4)$ & $12(2.9)$ & $66(15.8)$ & $15(3.6)$ \\
\hline Understanding maxillofacial pain & $105(25.1)$ & $152(36.3)$ & $52(12.4)$ & $15(3.6)$ & $78(18.6)$ & $17(4.1)$ \\
\hline Handling laser equipment & $140(33.4)$ & $152(36.3)$ & $28(6.7)$ & $9(2.1)$ & $70(16.7)$ & $20(4.8)$ \\
\hline
\end{tabular}

TMD: temporomandibular disorde. 


\section{Results}

With respect to jobs related to dental treatment, more than half of the respondents were able to perform their jobs, including beginners (Table 2). With regards to radiography tasks, $55.4 \%$ of the dentists answered that reading radiography was not the job of a dental hygienist and more than half of the dentists answered that radiography jobs, except special patients, could be done by beginners (Table 3 ). With regards to preventive treatments, more than half answered that most of the tasks could be performed by beginners except managing eating or swallowing disorders (43.2\%), managing multiple dental caries $(39.9 \%)$, managing implants $(32.0 \%)$, and oral hygiene of special patients (35.6\%) (Table 4). Between 12.2 to $18.6 \%$ of the dentists answered that all tasks in oral medicine could not be performed by a dental hygienist except for a hot pack (Table 5). In the case of root planning, $34.1 \%$ of respondents answered that the dental hygienists with more than 3 years of experience could perform the task, while $27.2 \%$ responded that it was not a task meant for dental hygienists (Table 6). With regards to the duties of conservative dentistry, $22.0 \%$ and $17.9 \%$ of the respondents responded that examination of pulp vitality and treatment for teeth whitening were not tasks for dental hygienists (Table 7). In prosthetic dentistry, 16.9 to $23.2 \%$ of the dentists responded that making individual trays, selecting tooth shades, and attaching and removing prostheses were not tasks meant for dental hygienists (Table 8). More than $10 \%$ of respondents responded that orthodontic diagnosis procedure, separating for band, and using elastics were not tasks meant for dental hygienists (Table 9). With regards to Oral and Maxillofacial Surgery (Table 10), $48.0 \%$ of dentists said

Table 6. Dentist's View of the Duties of Dental Hygienists in Periodontal Treatment

\begin{tabular}{|c|c|c|c|c|c|c|}
\hline \multirow[b]{2}{*}{ Duty } & \multicolumn{3}{|c|}{ Clinical experience required } & \multirow[b]{2}{*}{$\begin{array}{l}\text { Possible in } \\
\text { the future }\end{array}$} & \multirow[b]{2}{*}{ Impossible } & \multirow[b]{2}{*}{ No response } \\
\hline & $\begin{array}{l}\text { Beginners } \\
\text { available }\end{array}$ & $\begin{array}{c}3 \text { years or } \\
\text { more }\end{array}$ & $\begin{array}{c}5 \text { years or } \\
\text { more }\end{array}$ & & & \\
\hline Managing individual oral hygiene & $275(65.6)$ & $103(24.6)$ & $12(2.9)$ & $2(0.5)$ & $15(3.6)$ & $12(2.9)$ \\
\hline Examination of periodontal pockets & $203(48.4)$ & $124(29.6)$ & $18(4.3)$ & $11(2.6)$ & $5(1.2)$ & $5(1.2)$ \\
\hline Root planing & $110(26.3)$ & $143(34.1)$ & $27(6.4)$ & $20(4.8)$ & $114(27.2)$ & $5(1.2)$ \\
\hline Managing periodontal patients & $187(44.6)$ & $172(41.1)$ & $24(5.7)$ & $3(0.7)$ & $26(6.2)$ & $7(1.7)$ \\
\hline Assisting periodontal treatment or surgery & $259(61.8)$ & $130(31.0)$ & $15(3.6)$ & $2(0.5)$ & $9(2.1)$ & $4(1.0)$ \\
\hline Application of periodontal pack & $192(45.8)$ & $148(35.3)$ & $12(2.9)$ & $5(1.2)$ & $55(13.1)$ & $7(1.7)$ \\
\hline
\end{tabular}

Table 7. Dentist's View of the Duties of Dental Hygienists in Conservative and Pediatric Dentistry

\begin{tabular}{|c|c|c|c|c|c|c|}
\hline \multirow[b]{2}{*}{ Duty } & \multicolumn{3}{|c|}{ Clinical experience required } & \multirow{2}{*}{$\begin{array}{l}\text { Possible in } \\
\text { the future }\end{array}$} & \multirow[b]{2}{*}{ Impossible } & \multirow[b]{2}{*}{ No response } \\
\hline & $\begin{array}{c}\text { Beginners } \\
\text { available }\end{array}$ & $\begin{array}{c}3 \text { years or } \\
\text { more }\end{array}$ & $\begin{array}{c}5 \text { years or } \\
\text { more }\end{array}$ & & & \\
\hline Rubber dam application & $251(59.9)$ & $103(24.6)$ & $11(2.6)$ & $6(1.4)$ & $42(10.0)$ & $6(1.4)$ \\
\hline Matrix application & $228(54.4)$ & $108(25.8)$ & $12(2.9)$ & $9(2.1)$ & $49(11.7)$ & $13(3.1)$ \\
\hline Temporary filling & $248(59.2)$ & $111(26.5)$ & $15(3.6)$ & $6(1.4)$ & $34(8.1)$ & $5(1.2)$ \\
\hline Assisting conservative treatment & $298(71.1)$ & $90(21.5)$ & $10(2.4)$ & $1(0.2)$ & $14(3.3)$ & $6(1.4)$ \\
\hline Examining pulp vitality & $182(43.4)$ & $118(28.2)$ & $15(3.6)$ & $7(1.7)$ & $92(22.0)$ & $5(1.2)$ \\
\hline Assisting endodontic treatment & $297(70.9)$ & $83(19.8)$ & $12(2.9)$ & $1(0.2)$ & $19(4.5)$ & $7(1.7)$ \\
\hline Teeth brightening & $137(32.7)$ & $158(37.7)$ & $33(7.9)$ & $9(2.1)$ & $75(17.9)$ & $7(1.7)$ \\
\hline Assisting extraction of deciduous teeth & $334(79.7)$ & $50(11.9)$ & $7(1.7)$ & $2(0.5)$ & $19(4.5)$ & $7(1.7)$ \\
\hline Assisting SS crown treatment & $291(69.5)$ & $90(21.5)$ & $13(3.1)$ & $2(0.5)$ & $17(4.1)$ & $6(1.4)$ \\
\hline Assisting window opening & $278(66.3)$ & $100(23.9)$ & $14(3.3)$ & $1(0.2)$ & $16(3.8)$ & $10(2.4)$ \\
\hline Explaining preventive orthodontic treatment & $237(56.6)$ & $133(31.7)$ & $21(5.0)$ & $4(1.0)$ & $17(4.1)$ & $7(1.7)$ \\
\hline Assisting control of pediatric patient behavior & $250(59.7)$ & $132(31.5)$ & $18(4.3)$ & $2(0.5)$ & $10(2.4)$ & $7(1.7)$ \\
\hline
\end{tabular}

SS: stainless steel. 
Table 8. Dentist's View of the Duties of Dental Hygienists in Prosthetic Treatment

\begin{tabular}{|c|c|c|c|c|c|c|}
\hline \multirow[b]{2}{*}{ Duty } & \multicolumn{3}{|c|}{ Clinical experience required } & \multirow[b]{2}{*}{$\begin{array}{l}\text { Possible in the } \\
\text { future }\end{array}$} & \multirow[b]{2}{*}{ Impossible } & \multirow[b]{2}{*}{ No response } \\
\hline & $\begin{array}{c}\text { Beginners } \\
\text { available }\end{array}$ & $\begin{array}{c}3 \text { years or } \\
\text { more }\end{array}$ & $\begin{array}{c}5 \text { years or } \\
\text { more }\end{array}$ & & & \\
\hline Gingival retraction & $194(46.3)$ & $172(41.1)$ & $15(3.6)$ & $5(1.2)$ & $29(6.9)$ & $4(1.0)$ \\
\hline Making temporary crown & $170(40.6)$ & $185(44.2)$ & $22(5.3)$ & $11(2.6)$ & $26(6.2)$ & $5(1.2)$ \\
\hline Assisting crown \& bridge treatment & $283(67.5)$ & $107(25.5)$ & $13(3.1)$ & $2(0.5)$ & $8(1.9)$ & $6(1.4)$ \\
\hline Assisting denture treatment & $268(64.0)$ & $114(27.2)$ & $20(4.8)$ & $1(0.2)$ & $8(1.9)$ & $8(1.9)$ \\
\hline Making individual tray & $117(27.9)$ & $174(41.5)$ & $31(7.4)$ & $14(3.3)$ & $77(18.4)$ & $6(1.4)$ \\
\hline Selecting tooth shade & $127(30.3)$ & $150(35.8)$ & $51(12.2)$ & $9(2.1)$ & $71(16.9)$ & $11(2.6)$ \\
\hline Cementation and removal of prostheses & $152(36.3)$ & $126(30.1)$ & $26(6.2)$ & $11(2.6)$ & $97(23.2)$ & $7(1.7)$ \\
\hline Managing prostheses & $210(50.1)$ & $150(35.8)$ & $26(6.2)$ & $6(1.4)$ & $17(4.1)$ & $10(2.4)$ \\
\hline
\end{tabular}

Table 9. Dentist's View of the Duties of Dental Hygienists in Orthodontic Treatment

\begin{tabular}{|c|c|c|c|c|c|c|}
\hline \multirow[b]{2}{*}{ Duty } & \multicolumn{3}{|c|}{ Clinical experience required } & \multirow{2}{*}{$\begin{array}{l}\text { Possible in } \\
\text { the future }\end{array}$} & \multirow[b]{2}{*}{ Impossible } & \multirow[b]{2}{*}{ No response } \\
\hline & $\begin{array}{l}\text { Beginners } \\
\text { available }\end{array}$ & $\begin{array}{c}3 \text { years or } \\
\text { more }\end{array}$ & $\begin{array}{c}5 \text { years or } \\
\text { more }\end{array}$ & & & \\
\hline Understanding orthodontic diagnosis process & $158(37.7)$ & $138(32.9)$ & $52(12.4)$ & $6(1.4)$ & $48(11.5)$ & $17(4.1)$ \\
\hline Photo taking for orthodontic patients & $231(55.1)$ & $130(31.0)$ & $22(5.3)$ & $1(0.2)$ & $19(4.5)$ & $16(3.8)$ \\
\hline Using elastic seperator & $166(39.6)$ & $139(33.2)$ & $21(5.0)$ & $5(1.2)$ & $69(16.5)$ & $19(4.5)$ \\
\hline Assisting orthodontic treatment & $237(56.6)$ & $126(30.1)$ & $19(4.5)$ & $3(0.7)$ & $20(4.8)$ & $14(3.3)$ \\
\hline Ligaturing & $148(35.3)$ & $181(43.2)$ & $30(7.2)$ & $4(1.0)$ & $40(9.5)$ & $16(3.8)$ \\
\hline Assisting mini-screw application & $238(56.8)$ & $126(30.1)$ & $19(4.5)$ & $3(0.7)$ & $18(4.3)$ & $15(3.6)$ \\
\hline Using elastics & $183(43.7)$ & $140(33.4)$ & $24(5.7)$ & $3(0.7)$ & $50(11.9)$ & $19(4.5)$ \\
\hline Managing orthodontic pliers & $286(68.3)$ & $92(22.0)$ & $13(3.1)$ & $1(0.2)$ & $13(3.1)$ & $4(1.0)$ \\
\hline Managing orthodontic patients & $241(57.5)$ & $126(30.1)$ & $20(4.8)$ & $3(0.7)$ & $14(3.3)$ & $15(3.6)$ \\
\hline Managing oral hygiene of orthodontic patients & $284(67.8)$ & $94(22.4)$ & $11(2.6)$ & $1(0.2)$ & $11(2.6)$ & $18(4.3)$ \\
\hline
\end{tabular}

Table 10. Dentist's View of the Duties of Dental Hygienists in Oral and Maxillofacial Surgery

\begin{tabular}{|c|c|c|c|c|c|c|}
\hline \multirow[b]{2}{*}{ Duty } & \multicolumn{3}{|c|}{ Clinical experience required } & \multirow{2}{*}{$\begin{array}{l}\text { Possible in } \\
\text { the future }\end{array}$} & \multirow[b]{2}{*}{ Impossible } & \multirow[b]{2}{*}{ No response } \\
\hline & $\begin{array}{l}\text { Beginners } \\
\text { available }\end{array}$ & $\begin{array}{c}3 \text { years or } \\
\text { more }\end{array}$ & $\begin{array}{c}5 \text { years or } \\
\text { more }\end{array}$ & & & \\
\hline Assisting tooth extraction & $376(89.7)$ & $34(8.1)$ & $3(0.7)$ & $0(0.0)$ & $2(0.5)$ & $4(1.0)$ \\
\hline Assisting biopsy & $262(62.5)$ & $105(25.1)$ & $14(3.3)$ & $0(0.0)$ & $26(6.2)$ & $12(2.9)$ \\
\hline Assisting oral and maxillofaical surgery & $263(62.8)$ & $129(30.8)$ & $15(3.6)$ & $0(0.0)$ & $7(1.7)$ & $5(1.2)$ \\
\hline Managing patients with cleft lip and palate & $127(30.3)$ & $169(40.3)$ & $49(11.7)$ & $11(2.6)$ & $45(10.7)$ & $18(4.3)$ \\
\hline Managing patients with jaw reconstruction & $113(27.0)$ & $154(36.8)$ & $70(16.7)$ & $15(3.6)$ & $51(12.2)$ & $16(3.8)$ \\
\hline Managing patients with oral cancer & $92(22.0)$ & $134(32.0)$ & $91(21.7)$ & $20(4.8)$ & $64(15.3)$ & $18(4.3)$ \\
\hline Suture and stitch out & $107(25.5)$ & 75 (17.9) & $15(3.6)$ & $14(3.3)$ & $201(48.0)$ & 7 (1.7) \\
\hline
\end{tabular}

that suturing and stitching out were not tasks meant for a dental hygienist. More than half of the respondents responded that all jobs related to implants are available for beginners (Table 11). Most respondents responded that precision impression and digital impression required more than three years of experience (Table 12). Meanwhile, $36.0 \%$ of the respondents stated that intramuscular injection was not their job, while the highest percentage of re- spondents stated that emergency treatment is possible over three years (Table 13). Most respondents stated that dental hygienists with more than three years of experience could claim national insurance and counsel patients, and those with more than 5 years of experience could manage employees in a dental clinic (Table 14). With regards to self-development jobs, the response rate that beginners could perform such duties was highest for one or more 
Table 11. Dentist's View of the Duties of Dental Hygienists in Implant Treatment

\begin{tabular}{|c|c|c|c|c|c|c|}
\hline \multirow[b]{2}{*}{ Duty } & \multicolumn{3}{|c|}{ Clinical experience required } & \multirow[b]{2}{*}{$\begin{array}{l}\text { Possible in } \\
\text { the future }\end{array}$} & \multirow[b]{2}{*}{ Impossible } & \multirow[b]{2}{*}{ No response } \\
\hline & $\begin{array}{l}\text { Beginners } \\
\text { available }\end{array}$ & $\begin{array}{c}3 \text { years or } \\
\text { more }\end{array}$ & $\begin{array}{c}5 \text { years or } \\
\text { more }\end{array}$ & & & \\
\hline Understanding implant procedure & $279(66.6)$ & $117(27.9)$ & $10(2.4)$ & $0(0.0)$ & $7(1.7)$ & $6(1.4)$ \\
\hline Assisting implant surgery & $270(64.4)$ & $130(31.0)$ & $13(3.1)$ & $0(0.0)$ & $2(0.5)$ & $4(1.0)$ \\
\hline Assisting bone augmentation & $217(51.8)$ & $172(41.1)$ & $17(4.1)$ & $2(0.5)$ & $6(1.4)$ & $5(1.2)$ \\
\hline Assisting implant prostheses & $235(56.1)$ & $154(36.8)$ & $22(5.3)$ & $0(0.0)$ & $4(1.0)$ & $4(1.0)$ \\
\hline Managing implant instrument & $233(55.6)$ & $158(37.7)$ & $21(5.0)$ & $0(0.0)$ & $3(0.7)$ & $4(1.0)$ \\
\hline Managing oral hygiene of implant patients & $232(55.4)$ & $157(37.5)$ & $15(3.6)$ & $1(0.2)$ & $8(1.9)$ & $6(1.4)$ \\
\hline
\end{tabular}

Table 12. Dentist's View of the Duties of Dental Hygienists in Impression Taking, Model Fabrication, and Cement Mixing

\begin{tabular}{|c|c|c|c|c|c|c|}
\hline \multirow[b]{2}{*}{ Duty } & \multicolumn{3}{|c|}{ Clinical experience required } & \multirow{2}{*}{$\begin{array}{l}\text { Possible in } \\
\text { the future }\end{array}$} & \multirow[b]{2}{*}{ Impossible } & \multirow[b]{2}{*}{ No response } \\
\hline & $\begin{array}{l}\text { Beginners } \\
\text { available }\end{array}$ & $\begin{array}{c}3 \text { years or } \\
\text { more }\end{array}$ & $\begin{array}{l}5 \text { years or } \\
\text { more }\end{array}$ & & & \\
\hline Bite registration & $195(46.5)$ & $162(38.7)$ & $14(3.3)$ & $3(0.7)$ & $41(9.8)$ & $4(1.0)$ \\
\hline Alginate impression & $305(72.8)$ & $93(22.2)$ & $10(2.4)$ & $2(0.5)$ & $6(1.4)$ & $3(0.7)$ \\
\hline Rubber impression & $129(30.8)$ & $198(47.3)$ & $29(6.9)$ & $5(1.2)$ & $53(12.6)$ & $5(1.2)$ \\
\hline Digital impression & $104(24.8)$ & $184(43.9)$ & $34(8.1)$ & $13(3.1)$ & $67(16.0)$ & $17(4.1)$ \\
\hline Mixing cement or filling material & $350(83.5)$ & $59(14.1)$ & $4(1.0)$ & $1(0.2)$ & $3(0.7)$ & $2(0.5)$ \\
\hline Model fabrication & $267(63.7)$ & $111(26.5)$ & $8(1.9)$ & $6(1.4)$ & $25(6.0)$ & $2(0.5)$ \\
\hline
\end{tabular}

Table 13. Dentist's View of the Duties of Dental Hygienists in Anesthesia, Injection etc.

\begin{tabular}{|c|c|c|c|c|c|c|}
\hline \multirow[b]{2}{*}{ Duty } & \multicolumn{3}{|c|}{ Clinical experience required } & \multirow{2}{*}{$\begin{array}{l}\text { Possible in } \\
\text { the future }\end{array}$} & \multirow[b]{2}{*}{ Impossible } & \multirow[b]{2}{*}{ No response } \\
\hline & $\begin{array}{l}\text { Beginners } \\
\text { available }\end{array}$ & $\begin{array}{c}3 \text { years or } \\
\text { more }\end{array}$ & $\begin{array}{l}5 \text { years or } \\
\text { more }\end{array}$ & & & \\
\hline Topical anesthesia & $330(78.8)$ & $46(11.0)$ & $9(2.1)$ & $3(0.7)$ & $27(6.4)$ & $4(1.0)$ \\
\hline Preparing local anesthesia & $368(87.8)$ & $35(8.4)$ & $5(1.2)$ & $2(0.5)$ & $7(1.7)$ & $2(0.5)$ \\
\hline Intramuscular injection & $103(24.6)$ & 99 (23.6) & $21(5.0)$ & $33(7.9)$ & $151(36.0)$ & $12(2.9)$ \\
\hline Dealing with emergency in dental clinic & $92(22.0)$ & $146(34.8)$ & $85(20.3)$ & $16(3.8)$ & $73(17.4)$ & $7(1.7)$ \\
\hline Dealing with dental emergency & $92(22.0)$ & $151(36.0)$ & $85(20.3)$ & $12(2.9)$ & $70(16.7)$ & $9(2.1)$ \\
\hline
\end{tabular}

years of experience (Table 15).

\section{Discussion}

The role of a dental hygienist was created for the purpose of preventing oral disease and promoting oral health care and education. However, in Korea, unlike in Europe or the United States, dental hygienists performed more dental assistance and dental clinic management duties $^{8,13)}$. Kim and $\mathrm{Kim}^{6}$, in a survey of 214 dental hygienists, found that the most common duty was dental treatment assistance. Choi et al. ${ }^{14)}$ surveyed 612 dental hygienists and found that their duties included, from most common to least, dental clinic management, oral disease prevention, patient consultation \& reception, and dental treatment assistance. Kim et al. ${ }^{15)}$ noted that the highest frequency of duties was dental clinic management in a survey of 144 dental hygienists, followed by preventive treatment, dental treatment assistance, and oral health education. Kwon and Lee ${ }^{16)}$ also reported that dental hygienists with more than 11 years of experience performed a lot of managerial duties, and those with 6 to 10 years of experience usually provided dental treatment assistance. In the same context as in their previous studies, this study included not only oral disease prevention and education as the original duties of dental hygienists but also the duties related to the clinical field.

The dentists who participated in this study stated that 
Table 14. Dentist's View of the Duties of Dental Hygienists in Management and Administration

\begin{tabular}{|c|c|c|c|c|c|c|}
\hline \multirow[b]{2}{*}{ Duty } & \multicolumn{3}{|c|}{ Clinical experience required } & \multirow{2}{*}{$\begin{array}{l}\text { Possible in } \\
\text { the future }\end{array}$} & \multirow[b]{2}{*}{ Impossible } & \multirow[b]{2}{*}{ No response } \\
\hline & $\begin{array}{l}\text { Beginners } \\
\text { available }\end{array}$ & $\begin{array}{l}3 \text { years or } \\
\text { more }\end{array}$ & $\begin{array}{c}5 \text { years or } \\
\text { more }\end{array}$ & & & \\
\hline National insurance claim & $120(28.6)$ & $189(45.1)$ & $87(20.8)$ & $4(1.0)$ & $14(3.3)$ & $5(1.2)$ \\
\hline Maintaining facilities & $246(58.7)$ & $111(26.5)$ & $22(5.3)$ & $3(0.7)$ & $34(8.1)$ & $3(0.7)$ \\
\hline Maintaining equipments & $245(58.5)$ & $110(26.3)$ & $25(6.0)$ & $4(1.0)$ & $32(7.6)$ & $3(0.7)$ \\
\hline Managing prothesis & $269(64.2)$ & $128(30.5)$ & $17(4.1)$ & $1(0.2)$ & $2(0.5)$ & $2(0.5)$ \\
\hline Managing materials & $289(69.0)$ & $110(26.3)$ & $15(3.6)$ & $1(0.2)$ & $2(0.5)$ & $2(0.5)$ \\
\hline Managing purchase & $273(65.2)$ & $122(29.1)$ & $18(4.3)$ & $1(0.2)$ & $2(0.5)$ & $3(0.7)$ \\
\hline Managing documents & $236(56.3)$ & $135(32.2)$ & $40(9.5)$ & $2(0.5)$ & $4(1.0)$ & $2(0.5)$ \\
\hline Infection control & $236(56.3)$ & $144(34.4)$ & $31(7.4)$ & $1(0.2)$ & $4(1.0)$ & $3(0.7)$ \\
\hline Patient consultation & $138(32.9)$ & $173(41.3)$ & $103(24.6)$ & $2(0.5)$ & $1(0.2)$ & $2(0.5)$ \\
\hline Clinic promotion & $169(40.3)$ & $135(32.2)$ & 79 (18.9) & $5(1.2)$ & $28(6.7)$ & $3(0.7)$ \\
\hline Managing employee & $100(23.9)$ & $110(26.3)$ & $188(44.9)$ & $3(0.7)$ & $14(3.3)$ & $4(1.0)$ \\
\hline
\end{tabular}

Table 15. Dentist's View of the Duties of Dental Hygienists in Self Development

\begin{tabular}{|c|c|c|c|c|c|c|}
\hline \multirow[b]{2}{*}{ Duty } & \multicolumn{3}{|c|}{ Clinical experience required } & \multirow{2}{*}{$\begin{array}{l}\text { Possible in the } \\
\text { future }\end{array}$} & \multirow[b]{2}{*}{ Impossible } & \multirow[b]{2}{*}{ No response } \\
\hline & $\begin{array}{l}\text { Beginners } \\
\text { available }\end{array}$ & $\begin{array}{c}3 \text { years or } \\
\text { more }\end{array}$ & $\begin{array}{l}5 \text { years or } \\
\text { more }\end{array}$ & & & \\
\hline Understanding professionalism & $238(56.8)$ & $118(28.2)$ & $48(11.5)$ & $5(1.2)$ & $6(1.4)$ & $4(1.0)$ \\
\hline Gaining new knowledge and skills & $257(61.3)$ & $105(25.1)$ & $44(10.5)$ & $3(0.7)$ & $6(1.4)$ & $4(1.0)$ \\
\hline Social contribution & $300(71.6)$ & $60(14.3)$ & $36(8.6)$ & $9(2.1)$ & $9(2.1)$ & $5(1.2)$ \\
\hline
\end{tabular}

most of the duties, from preventive treatment to dental treatment assistance, are can be performed by dental hygienists. In some cases, the current legal duties of a dental technician, radiologist, or physiotherapist were recognized as equal to that of a dental hygienist work. Many respondents recognized that dental computed tomography, digital radiography, and oral scanners should be performed by dental hygienists. The dentists thought that dental hygienists should oversee dental laboratories, radiography, and physical therapy in the dental clinic. Lee et al. ${ }^{3)}$ said that the need for range expansion of dental hygienists' work was agreed by $91.1 \%$ of dentists and $95.5 \%$ of dental hygienists. As for the detailed duty items that need to be expanded, both dentists and dental hygienists responded in the order of temporary crown making and setting, surgery assistance, and dental treatment assistance. Han et al. ${ }^{17)}$ noted that some dentists insisted that the current legal practice of dental hygienists does not fit the time and tasks that can be performed through education and training are often excluded from legal work.

The duties of dental hygienists not perceived by the dentists were reading radiography $(55.4 \%)$, suture and stich out $(48.0 \%)$, intramuscular injection $(36.0 \%)$, root planning $(27.2 \%)$, cementation and removal of prostheses $(23.2 \%)$, examining pulp vitality $(22.0 \%)$, etc. In a study by Hwang et al. ${ }^{18)}$, the tasks considered by dentists not to be the duty of dental hygienists included infiltration anesthesia investigation, filling in cavities, intramuscular injection, canal irrigation, setting crown and bridge, and making individual tray. This result is similar to our survey.

Although this study has a limitation of convenience extraction that does not consider the area or age group, it can be said that this study has a significant number of dentists who surveyed opinions on the duties of dental hygienists. In this study, there were certain tasks whereby the opinions of the dentists were different; however, most of the tasks were thought could be performed by dental hygienists. Hwang and $\mathrm{Han}^{19)}$ stated that it is necessary for the law to include the duties that many dental hygienists perform under the supervision of a dentist. Han et al. ${ }^{17)}$ insisted that the law should state the duties that dental hygienists should not do, not those which they could do. 
Considering the rapidly changing dental equipment and the contribution of dental hygienists in the field, a more careful listing of the legal duties of dental hygienists is expected to be amended. Therefore, there is a need for flexibility when such amendments are made, especially for dental hygienists who can keep up with the rapid changes in dental technology.

\section{Notes}

\section{Conflict of interest}

No potential conflict of interest relevant to this article was reported.

\section{Ethical approval}

This study was approved by the Institutional Bioethics Committee of Daejeon Health Institute of Technology in 2017 (IRB no. 1041490-20170118-HR-001).

\section{Author contributions}

Conceptualization: Yang-Keum Han. Data acquisition: Yang-Keum Han, Ji-Su Yu, Seung-Hee Kim, Jin-Young Yang, Soo-Myoung Bae, Soo-Jeong Hwang. Formal analysis: Ji-Su Yu, Soo-Jeong Hwang. Funding: Korea Dental Hygienists Association. Supervision: Yang-Keum Han. Writing-original draft: Ji-Su Yu, Soo-Jeong Hwang. Writing-review \& editing: Yang-Keum Han, Ji-Su Yu, Seung-Hee Kim, Jin-Young Yang, Soo-Myoung Bae, Soo-Jeong Hwang.

\section{ORCID}

Yang-Keum Han, https://orcid.org/0000-0002-5074-0053 Ji-Su Yu, https://orcid.org/0000-0003-4571-6988 Seung-Hee Kim, https://orcid.org/0000-0003-2425-8965 Jin-Young Yang, https://orcid.org/0000-0002-5655-4438 Soo-Myoung Bae, https://orcid.org/0000-0002-1802-4129 Soo-Jeong Hwang, https://orcid.org/0000-0003-4725-1512

\section{Acknowledgements}

This research was supported by Korea Dental Hygienists Association in 2017.

We appreciate Professor Eung-Kwon Kim at Suwon Science College surveying the opinions of dentists in metropolitan area.

\section{References}

1. Cho YS: Comparison of curriculums of dental hygiene education programs for B. S degree. J Dent Hyg Sci 5: 251-258, 2005.

2. Cho YS, Lee SY: Developing a integrated curriculum for a clinical dental hygiene. J Dent Hyg Sci 5: 33-38, 2005.

3. Lee HJ, Shin SJ, Bae SM, Shin BM: A survey of dentists and dental hygienists on dental team composition and work assignments. J Korean Dent Hyg Sci 2: 51-62, 2019.

4. Gwon MY, Jung MH: Study of dental hygienist's stress degree and adaptation method. J Dent Hyg Sci 4: 133-140, 2004.

5. Kim YS, Shin MW: A study on the current state and weight of dental hygienists' works. J Korean Soc Dent Hyg 8: 161-175, 2008.

6. Kim JH, Kim HJ: Relationship between turnover and job stress of dental hygienists. J Dent Hyg Sci 11: 47-53, 2011.

7. Yoo EM, Han HJ: A study on the work and turnover of clinical dental hygienists. J Dent Hyg Sci 11: 41-46, 2011.

8. Park JH, Kim MS, Cho JW: Dentists' opinions for dental hygienists' roles in Korea. J Korean Acad Oral Health 34: 88-97, 2010

9. Lim MH, Han SJ, Chung JY: A study on dentists' awareness about dental hygienists' works and duty division. J Korean Acad Dent Hyg 6: 71-86, 2004.

10. Lee SY, Yu BC: Relationship between occupational stress and work-related factors in dental hygienists. J Dent Hyg Sci 11: 553-562, 2011.

11. Park GE, Lee YM, Lee JG, Jeon HS: A survey on dental college students' perception and understanding of dental hygienists. J Korean Soc Dent Hyg 15: 531-538, 2015.

12. Park JL, Kang KH, Kin SA, et al.: The 2 nd job analysis of dental hygienists. Retrieved January 10, 2020, from https:// rnd.kuksiwon.or.kr/last/selectLastDetail.do?MENU_ID $=\mathrm{C}-0$ $1-01 \&$ reportno $=$ RE02-1204-15\&sYear $=\&$ sJssfc_code $=12 \& \mathrm{~s}$ Researchindexcd $=\&$ researcherid $=\&$ sRsrchtasknm $=\&$ PAGE _NUM=1\&PER_PAGE=10\&IS_PAGE_NEW_SEARCH= Y\&TOTAL_PAGE=8(2012).

13. Luciak-Donsberger $C$ : Origins and benefits of dental hygiene practice in Europe. Int J Dent Hyg 1: 29-42, 2003. 
https://doi.org/10.1034/j.1601-5037.2003.00008.x

14. Choi HN, Bae HS, Cho YS: Effect of gap between expected job and performed job on turnover intention and job satisfaction in clinical dental hygienists. J Dent Hyg Sci 11: 463-469, 2011.

15. Kim EG, Lim SH, Kwon MY, Choi YY, Han JH: Analysis of tasks and education needs for dental hygienist for development of dental hygiene curriculum. J Dent Hyg Sci 14: 35-42, 2014.

16. Kwon JY, Lee SY: Relationship of between task performance, job satisfaction, and organizational contribution of dental hygienists. J Dent Hyg Sci 16: 302-309, 2016. https://doi.org/10.17135/jdhs.2016.16.4.302

17. Han YK, Kim SH, Yang JY, Yu JS, Bae SM: A survey of dentists' opinions on the performance of dental hygienists. J Korean Acad Dent Admin 7: 1-9, 2019.

18. Hwang SJ, Koong HS, Lee SH: Difference of perception of the duties of dental hygienist between dentists and dental hygiene students in an area. J Korean Acad Dent Admin 5: 1-12, 2017.

19. Hwang SJ, Han YK: Frequency and time of actual duties of some dental hygienists. J Korean Acad Dent Admin 7: 10-20, 2019. 